\section{Hydrogel-based platforms to mimic in vivo drug diffusion: A multicenter research}

\author{
Paola Petrini, ${ }^{1}$ Daniela Pacheco, ${ }^{1}$ \\ Francesco Briatico Vangosa, ${ }^{1}$ \\ Cosmin S. Butnarasu, ${ }^{2}$ Livia Visai, $, 3,4$ \\ Orietta Monticelli, ${ }^{5}$ Laura Pastorino, ${ }^{6}$ \\ Sonja Visentin² \\ 1Department of Chemistry, Materials \\ and Chemical Engineering "G. Natta", \\ Politecnico di Milano, Milan; 2 Molecular \\ Biotechnology and Health Sciences \\ Department, University of Torino, \\ Turin; ${ }^{3}$ Department of Molecular \\ Medicine, Center for Health \\ Technologies (CHT), University of \\ Pavia, Pavia; ${ }^{4}$ Department of \\ Occupational Medicine, Toxicology and \\ Environmental Risks, S. Maugeri \\ Foundation, IRCCS, Pavia; \\ 5Department of Chemistry and \\ Industrial Chemistry, University of \\ Genoa, Genoa, Italy; 'Department of \\ Informatics, Bioengineering, Robotics \\ and System Engineering, University of \\ Genoa, Genoa, Italy
}

\begin{abstract}
An airway mucus model is proposed thus serving as an in vitro screening tool with the aim to reduce the number of noneffective drugs reaching the preclinical trials. The engineered mucus model is an easy-to-use and easy-to-produce tool that can be easily coupled to state-of-art diffusion models and it is compatible with high throughput analysis. This platform will serve as the basis to implement the complexity of the model in terms of components, also including the effect of bacteria.
\end{abstract}

\section{Introduction}

Mucus is a natural barrier of all organs exhibiting an air-liquid interface, which selectively filters the passage of gases, pathogens, pollutants, and nutrients, but also a steric and interactive barrier for drug permeability.1,2 Mucus also hosts particular microbial niches that co-exist in symbiosis or dysbiosis within the human body, yet the consequences of the latter are only now being significantly addressed mainly in animal testing and from the clinical point of view. In diseases like cystic fibrosis (CF), mucus exhibits altered physicochemical properties, which hamper clearance mechanisms and limits drug diffusion. The inefficient mucus secretion enables the establishment of bacterial colonies that trigger chronic infection, and lately lead to lung failure. New strategies are developed to overcome mucus barrier. ${ }^{3,4}$ The need to characterize drug permeability in a rapid, simple and reproducible manner has urged the development of mucus models. ${ }^{5}$ As the full reproduction of the complexity of the mucus barrier is an ambitious task, an airway mucus model is herein proposed to disassemble the complexity of the mucus barrier following a modular approach.

\section{Materials and Methods}

Alginate (alginic acid sodium salt, from brown algae)/mucin (from porcine stomach, type III) hydrogels were developed taking advantage of the internal crosslinking mechanism of alginate, in the presence of $\mathrm{NaCl}$ (final concentration $7 \mathrm{mM}$ ). Rheological measurements were carried out to access the viscoelastic and shear thinning behaviour of the developed gels and further compared to the pathological CF-mucus. Stability analysis was also conducted to acquire using both water and PBS, at $25^{\circ} \mathrm{C}$, to analyse changes in weight percentage and volumetric increase. Finally, both drug diffusion and interaction through alginate and alginate/mucin gels were carried out using aspirin, cephalexin, and epirubicin, as well as gold nanoparticles (GNP) as model drugs.

\section{Results}

The steric retention of CF mucus was reproduced by targeting its mesh size (approximately $50 \mathrm{~nm}$ ) and rheological properties, while the interactive barrier was reproduced by adopting a composition inspired by the CF mucus. The mucus models, mainly composed of mucin in an alginate (Alg) hydrogels, were developed aiming at specifically modelling the chemicalphysical properties of $\mathrm{CF}$ mucus. A combined set of experimental and mathematical techniques was developed and applied to target similar viscoelastic properties as those reported for $\mathrm{CF}$ sputum at both breathing and mucociliary beating frequencies. Optimized mucus models, composed of $3 \mathrm{mg} / \mathrm{mL} \mathrm{Alg}$ and $25 \mathrm{mg} / \mathrm{mL}$ mucin, exhibited a $G^{\prime}$ increasing from $\sim 21.2$ to $55.2 \mathrm{~Pa}$ and $\mathrm{G}$ " ranging from $\sim 5.26$ to 28.8 $\mathrm{Pa}$ in the frequency range of 0.1 to $20 \mathrm{~Hz}$. Stability analysis in water, PBS, and $\mathrm{DMSO}$, at $25^{\circ} \mathrm{C}$, revealed an increased
Correspondence: Correspondence: Paola Petrini, Department of Chemistry, Materials and Chemical Engineering "G. Natta", Politecnico di Milano, Milan, Italy.

E-mail: paola.petrini@polimi.it

Key words: In vitro models; diffusion profiles; hydrogels; mucins; permeability.

Conference presentation: this paper was presented at the Second Centro 3R Annual Meeting - 3Rs in Italian Universities, 2019 , June 20-21, University of Genoa, Italy.

Received for publication: 28 October 2019. Accepted for publication: 6 November 2019.

This work is licensed under a Creative Commons Attribution NonCommercial 4.0 License (CC BY-NC 4.0).

(C) Copyright: the Author(s), 2019

Licensee PAGEPress, Italy

Biomedical Science and Engineering 2019; 3(s2):101 doi:10.4081/bse.2019.101

weight and thickness mainly in the early hours. Diffusion studies of three model drugs through the mucus models unveil the ability of the mucus model to discriminate their mucin-drug interaction and the steric barrier of a mucus layer with respect to PAMPA that models the phospholipidic cell membrane, the state-of-art screening tool for passive drug diffusion.

\section{Conclusions}

The mucus model is proposed as an in vitro tool for early drug discovery, representing a step forward on modelling the mucus layer that is often not considered when assessing drug permeability. The engineered mucus model is an easy-to-use and easy-to-produce tool that can be easily coupled to state-of-art diffusion models (e.g. PAMPA membranes) and it is compatible with high throughput analysis. Finally, the production method allows to easily incorporating, in a modular approach, other mucus components (e.g. albumin, phospholipids, among others) to further recreate the chemical composition of mucus, while allowing to distinguish the contribution of each component over drug permeability. This platform will serve as the basis to implement the complexity of the model in terms of components, also including the effect of bacteria. 
The case of theophylline, prednisolone and cephalexin. Bioorg Med Chem 2015;23:6581-6.

3. Boi S, Dellacasa E, Bianchini P, et al. Encapsulated functionalized stereocomplex PLA particles: An effective system to support mucolytic enzymes. Colloids Surfaces B: Biointerfaces 2019;179: 190-8.

4. Butnarasu C, Barbero N, Pacheco D, et al. Mucin binding to therapeutic molecules: The case of antimicrobial agents used in cystic fibrosis. Int J Pharm 2019;564:136-44.

5. Peneda Pacheco D, Butnarasu C.S., Briatico Vangosa F., et al. Disassembling the complexity of mucus barriers to develop a fast screening tool for early drug discovery. J Mater Chem B. 2019 Aug 14;7(32):4940-4952. 